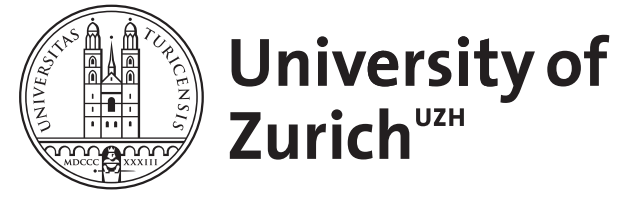
Archive

University of Zurich

University Library

Strickhofstrasse 39

CH-8057 Zurich

www.zora.uzh.ch

Year: 2009

\title{
Pitfalls in paediatric musculoskeletal imaging
}

Kellenberger, C J

DOI: https://doi.org/10.1007/s00247-009-1220-y

Posted at the Zurich Open Repository and Archive, University of Zurich

ZORA URL: https://doi.org/10.5167/uzh-30530

Journal Article

Published Version

Originally published at:

Kellenberger, C J (2009). Pitfalls in paediatric musculoskeletal imaging. Pediatric Radiology, 39(Suppl):372381.

DOI: https://doi.org/10.1007/s00247-009-1220-y 


\title{
Pitfalls in paediatric musculoskeletal imaging
}

\author{
Christian J. Kellenberger
}

(C) Springer-Verlag 2009

\section{Introduction}

Many pitfalls in paediatric musculoskeletal imaging are related to misleading history or clinical findings. There is often a history of antecedent minor trauma in children with musculoskeletal symptoms that are not necessarily the cause of a detected lesion (e.g. in patients with osteomyelitis or tumours). Alternatively, trauma may not be obvious or has been forgotten, preventing its inclusion in the differential diagnosis. The area of pathology may not be imaged, because pain is referred to another anatomical site (e.g. knee pain when there is hip pathology). In a limping child, localising symptoms can be absent, so that pathology may be missed if the entire lower extremities, pelvis and spine are not investigated. Imaging findings themselves may be misleading, either by being non-specific or by mimicking another entity. MRI has become an important modality for the evaluation of musculoskeletal disorders, but it also has the potential for adding confusion due to the non-specific findings in many cases. This review covers some of the normal variants, developmental changes and pathological lesions of soft tissue and bone that can lead to interpretation pitfalls in children, with emphasis on their MRI appearances.
C. J. Kellenberger $(\bowtie)$

Department of Diagnostic Imaging,

University Children's Hospital,

Steinwiesstrasse 72 ,

CH 8032 Zürich, Switzerland

e-mail: christian.kellenberger@kispi.uzh.ch

\section{Anatomical variants and developmental lesions}

Numerous anatomical variants and developmental changes in the growing skeleton can pose diagnostic challenges as they may simulate lesions caused by disease. Irregularities of mineralisation and ossification occur normally in several epiphyses, apophyses and at the bony junctions adjacent to the growth plate (Figs. 1 and 2). The common sites of such normal bony irregularities, locations of accessory ossification centres and bones, and other skeletal variations are well described. Routine consultation of the textbooks by Caffey [1], Keats [2] or Köhler and Zimmer [3] is helpful to avoid the misinterpretation of a normal variant as pathology.

In our practice, the commonly recognised cortical irregularity of the femur due to avulsion frequently leads to concerns of underlying osteomyelitis or a malignant lesion, especially among referring physicians. The location at the insertion of the medial head of the gastrocnemius or adductor magnus muscle and typical appearance of a lytic lesion with sharp and often sclerotic margins should allow a clear radiographic diagnosis (Fig. 2). Avulsive cortical irregularities are usually asymptomatic and are not responsible for the patients' symptoms. On MRI, these lesions show isointense signal on $\mathrm{T} 1-\mathrm{W}$ sequences compared to muscle, hyperintense signal on fluid-sensitive sequences, and often contrast enhancement [4-6]. Adjacent oedemalike bone marrow changes have been described and are believed to be related to chronic stress reaction [7], but usually the surrounding soft tissues and bone marrow demonstrate normal signal.

Fibrous cortical defects (non-ossifying fibromas), the most common benign bone tumours encountered during childhood and adolescence, are usually discovered incidentally at the metadiaphysis of the long bones in the lower extremities. 


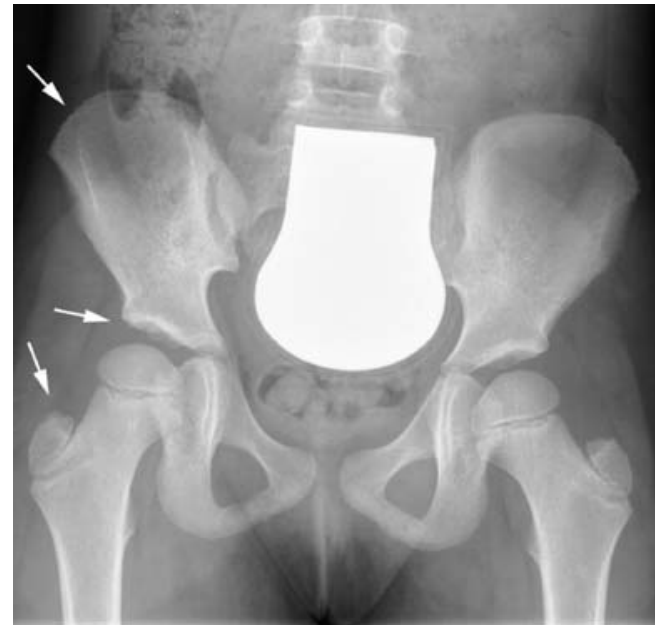

Fig. 1 Frontal pelvic radiograph of a 6-year-old girl shows normal irregular ossification of the right pelvic rim, acetabulum and greater trochanter (arrows)

Their characteristic plain film findings of a slightly expansile, cortically based lytic lesion with well-defined and often sclerotic borders should allow a definite diagnosis and obviate further imaging or even biopsy. On MRI, fibrous cortical defects demonstrate a hypointense rim, central low signal on T1-W images, variable signal on fluid-sensitive sequences, and often strong enhancement [8].

Most anatomical variants found in asymptomatic children are of no clinical significance and their recognition can prevent unnecessary studies and anxiety. However, some variants may indeed be clinically significant causing pain, most likely due to repetitive trauma or mechanical stress [9]. Examples of such potentially significant variants are bipartite patella, dorsal defect of the patella, os tibiale externum, os trigonum (Fig. 3) and fragmentation of the tibial tuberosity (Osgood-Schlatter disease) or calcaneal apophysis (Sever disease). The diagnosis of a symptomatic anatomical variant is usually made clinically when there is focal pain and tenderness, but can be supported by bone scintigraphy demonstrating increased radionuclide uptake, or MRI with oedema-like signal changes and increased contrast enhancement in the marrow of the affected bone and surrounding soft tissues.

The ischiopubic synchondrosis usually closes asymmetrically between the age of 4 and 12 years and can present as unilateral swelling with irregular mineralisation on radiographic examination. This normal ossification pattern of the synchondrosis should not be mistaken for a destructive lesion, but it can be associated with pain believed to arise from motion produced by the muscular insertions in the region around the synchondrosis. Even in asymptomatic children, the ischiopubic synchondrosis frequently shows increased radionuclide uptake on bone scintigraphy as well as oedema-like signal changes and contrast enhancement in the adjacent bone marrow and soft tissues on MRI [10-12], and therefore should not be confused with pathology (Fig. 4). However, in symptomatic patients, MRI of the entire pelvis is valuable for excluding pathology at other sites or diagnosing osteomyelitis originating from the metaphyseal equivalents of the ischium or pubis by showing true bone destruction, extensive soft tissue changes or an abscess [13].

\section{Bone marrow}

\section{Developmental changes}

The normal developmental changes of the bone marrow that occur during skeletal maturation need to be understood in order to be able to differentiate them from disease [14-16]. In the neonate, the entire bone marrow is haematopoietic, with low signal intensity on T1-W images (hypo- to isointense relative to muscle) and intermediate signal intensity on fluid-sensitive sequences, such as short inversion time inversion recovery (STIR) or fat-saturated T2-W fast spin-echo (isointense to slightly hyperintense relative to muscle). Because haematopoietic marrow is highly vascularised, its signal will increase following the administration of intravenous contrast material $[17,18]$.

Conversion from haematopoietic red marrow to yellow fatty marrow begins during the first year of life in the appendicular skeleton and progresses centrally until early adulthood [14]. With an increasing amount of fatty marrow, signal intensity gradually increases on $\mathrm{T} 1-\mathrm{W}$ images and decreases on fluid-sensitive sequences. By the end of the first year of life, the bone marrow of the hands and feet is usually completely transformed to yellow marrow with isointense signal relative to subcutaneous tissue [19]. However, in a large percentage of asymptomatic children multiple scattered small foci or more extensive areas of high signal may be seen in the foot and ankle on fluidsensitive sequences (Fig. 3). These foci are believed to represent persisting perivascular islands of red marrow and should not necessarily be interpreted as oedema following trauma or altered weight bearing $[20,21]$.

Epiphyseal and apophyseal ossification centres initially contain red marrow, which is transformed to yellow marrow within a few months of their appearance [22]. In the long bones of the extremities, transformation to yellow marrow progresses from the periphery towards the proximally placed bones, starting in the diaphyses and gradually expanding to the distal and proximal metaphyses [23-25]. Haematopoietic marrow can persist in the metaphyses as flame-shaped, geographic, or more ill-defined foci of red marrow with intermediate signal intensity (hyperintense relative to muscle on T1-W images) (Fig. 5). A degree of 

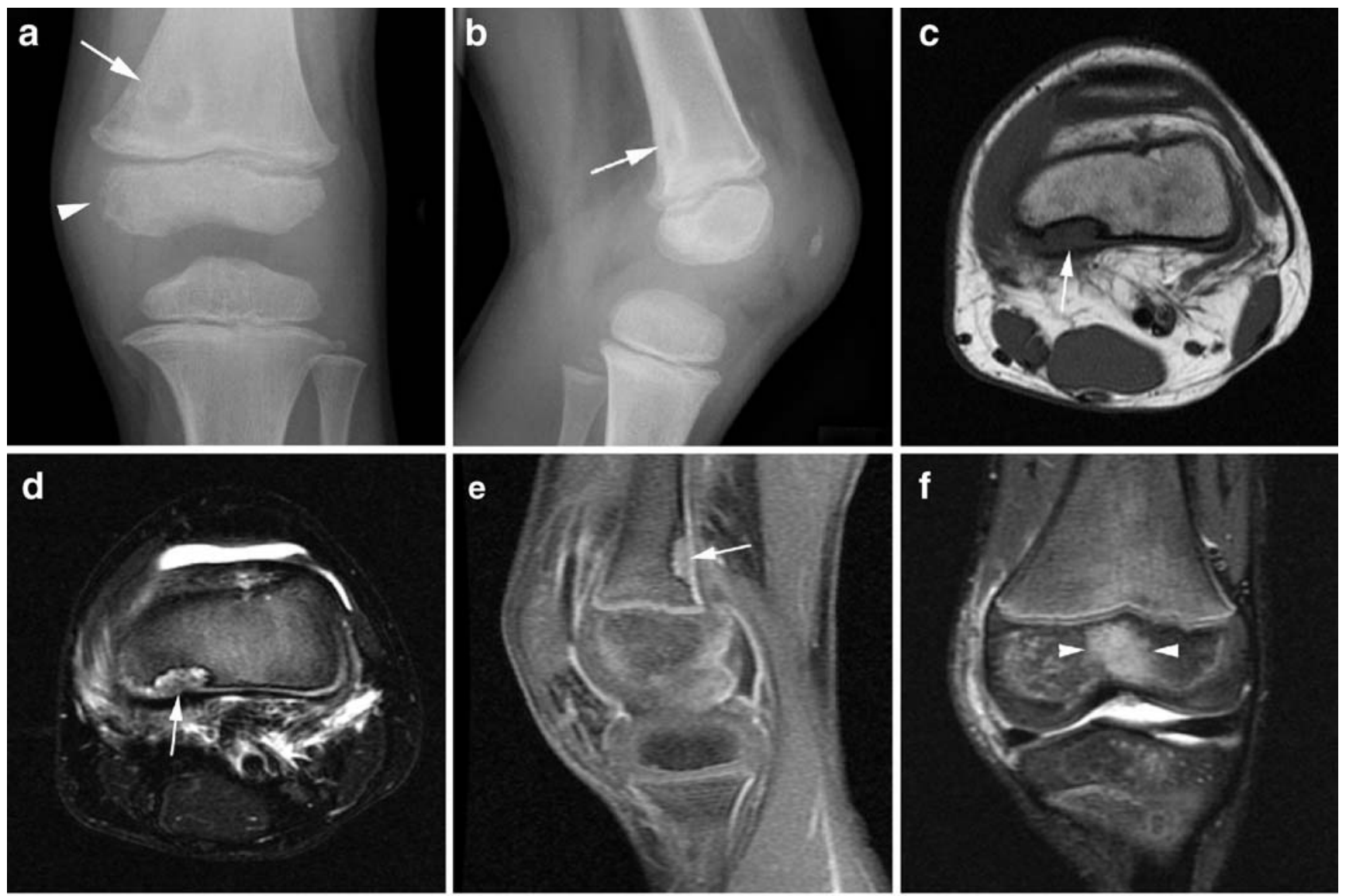

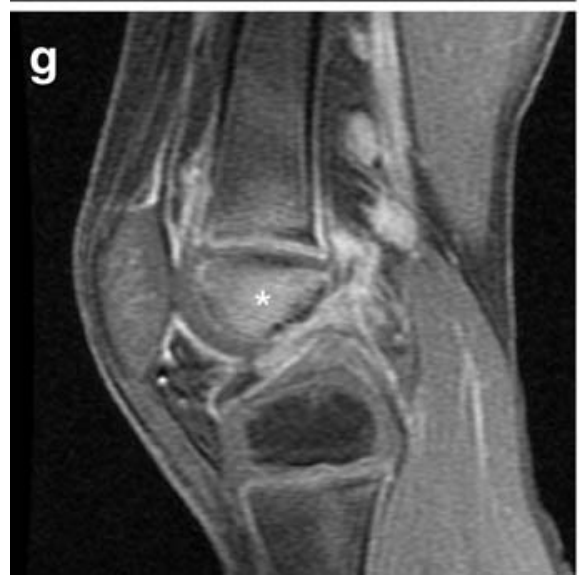

Fig. 2 A 4-year-old boy with a history of minor trauma, left knee pain and fever. a, b Radiographs of the left knee show a well-defined lytic lesion (arrows) at the posterior margin of the medial metaphysis, characteristic for avulsive cortical irregularity of the femur. In addition, the femoral ossification centre shows normal irregular ossification (arrowhead). c-e MRI of the left knee confirms avulsive cortical irregularity (arrows) at the origin of the medial head of the gastrocnemius muscle. The internal signal characteristics are isointense to muscle on T1-W images (c), hyperintense on T2-W images

red marrow can normally be seen in the proximal humeral and femoral metaphyses into early adulthood.

In the axial skeleton, marrow conversion occurs continuously with a steady increase in the proportion of fatty marrow. with fat saturation (d), and homogenous contrast-enhancement on T1W with fat saturation (e). The child's symptoms were due to bloodculture-proven bacterial osteomyelitis involving the femoral epiphysis. The coronal STIR image (f) shows focal non-specific oedema-like signal alteration (arrowheads) while the sagittal T1-W image with fat saturation demonstrates diffuse enhancement (asterisk) (g). Note additional reactive arthritis (negative joint aspiration) with a small effusion, prominent synovial enhancement and soft tissue oedema

On T1-W images, the signal of the vertebrae relative to the intervertebral discs is usually hypointense in the first year of life, isointense or slightly hyperintense between 1 and 5 years of age and hyperintense thereafter [26]. On fluid-sensitive 
Fig. 3 A 16-year-old boy with posterior ankle pain. a Lateral radiograph of the ankle shows an os trigonum (arrow). b Sagittal STIR MRI demonstrates increased bone marrow signal (arrow) indicating that an irritated os trigonum is responsible for the symptoms. The scattered small foci of hyperintense signal in the calcaneus (arrowheads) are a normal finding
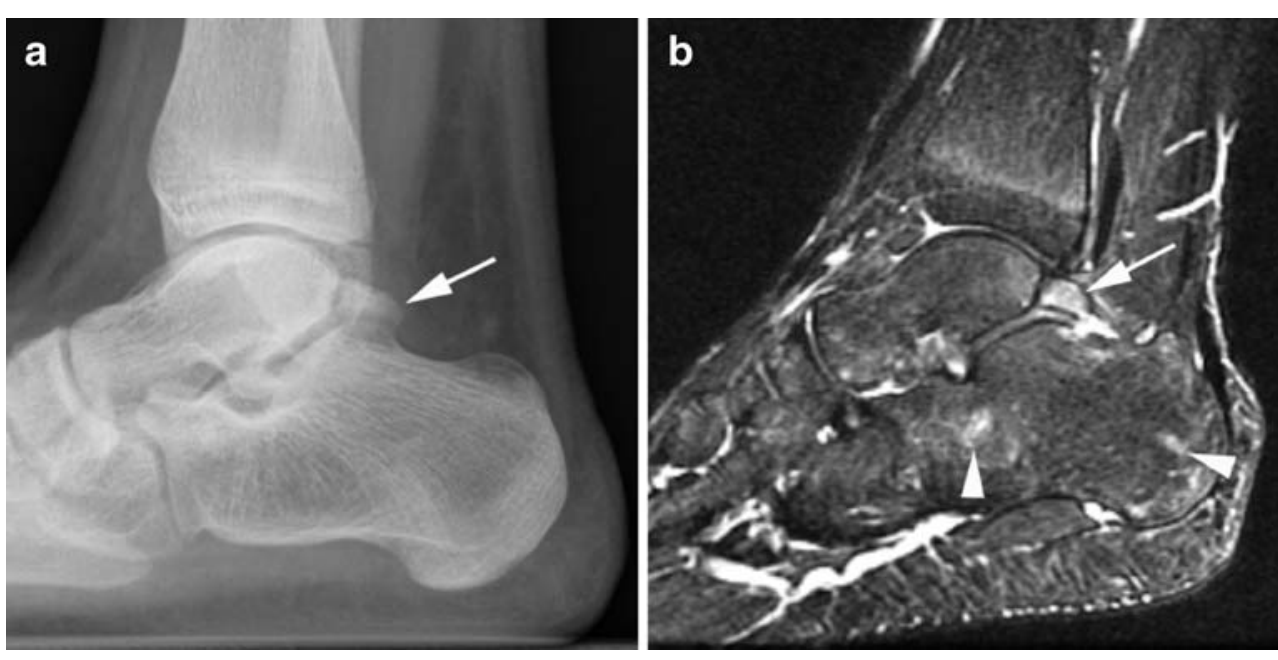

sequences with fat saturation (STIR, T2-W with spectral fat saturation), the vertebral signal relative to muscle gradually decreases from slightly hyperintense to isointense and eventually becomes hypointense in older children. The marrow signal of the pelvis in general parallels that of the spine, but it may be rather heterogeneous with areas containing a higher proportion of red marrow adjacent to areas with more fatty marrow [27]. In our experience, the sternum usually maintains a homogenous hyperintense signal relative to muscle on STIR images, which is often of higher intensity than that of the vertebral bodies and potentially could be mistaken for disease.

\section{Pathological conditions}

Diffuse bone marrow pathology, such as infiltration by malignant cells in leukaemia, lymphoma and sarcomas, as well as red marrow hyperplasia in various anaemias and after treatment with granulocyte colony stimulating factor, results in homogeneous low marrow signal isointense to muscle on T1-W images and marked hyperintensity on fluid-sensitive sequences (Fig. 6). Reconversion of yellow to red marrow begins in the axial skeleton and then extends to the appendicular skeleton, in a pattern opposite to normal marrow conversion [15]. Marrow involvement by lymphoma, metastases from neuroblastoma or Ewing sarcoma, and other malignancies metastasising to bone more often demonstrate multiple focal lesions than diffuse infiltration, which are preferentially located at sites with well vascularised red marrow.

A focal marrow lesion with very low signal on T1-W images, hyperintense signal relative to muscle on fat-saturated fluid-sensitive sequences and increased contrast enhancement is suspicious for marrow disease, but is entirely non-specific. Such oedema-like signal alterations result from increased water content due to cellular infiltration, marrow oedema, haemorrhage, hyperaemia, necrosis, and even fibrosis [28]. Additional findings need to be present in order to diagnose
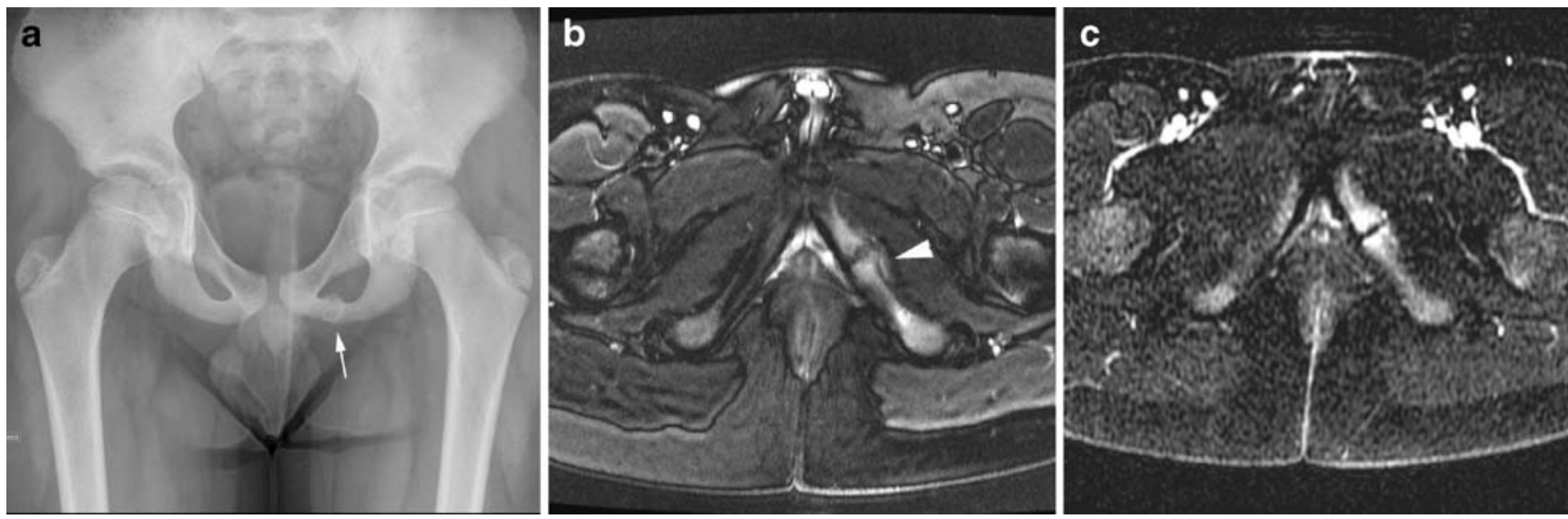

Fig. 4 A 9-year-old boy with chronic pain in the left groin. a Frontal radiograph shows a widened, irregularly mineralised left ischiopubic synchondrosis. b Axial T2-W fat-saturated MRI shows increased signal in the adjacent bone marrow of the pubis and ischium (arrowhead). c Axial dynamic gradient-echo MRI shows strong early contrast enhancement. These findings may be normal, occurring around the time of synchondrosal closure 

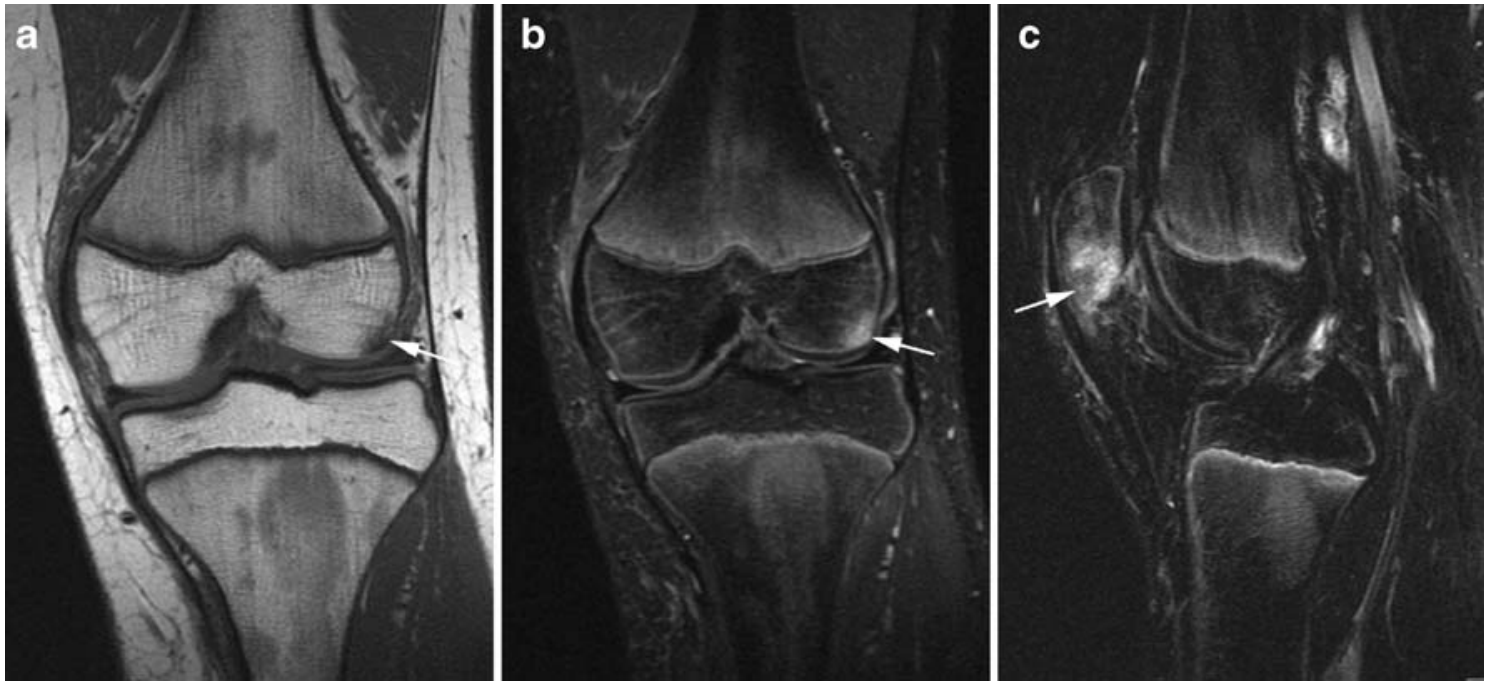

Fig. 5 MR images of a 12-year-old boy following left patellar dislocation. a Coronal T1-W and b STIR images show patchy areas of normal residual red marrow in the metaphyses with slightly hyperintense signal relative to muscle. Bone bruises at typical

the underlying aetiology on MRI. Osseous destruction with disruption of trabeculae and cortical bone, periosteal reaction, soft-tissue mass and oedema are features of malignant bone tumours but also of osteomyelitis and Langerhans cell histiocytosis. Identification of characteristic features of osteomyelitis on MRI, such as non-enhancing soft tissue or intraosseous abscesses, cloacae and sinus tracts, may allow exclusion of a bone tumour [29]. Acute-phase early lesions in Langerhans cell histiocytosis may appear very aggressive with cortical disruption and an accompanying soft tissue mass (Fig. 7), hence biopsy is required for diagnosis and differentiation from malignant lesions or osteomyelitis [30]. locations in the lateral femoral condyle and patella (arrows) show higher signal than red marrow on STIR and on the sagittal T2-W fatsaturated image (c)

In any destructive lesion, correlation of MRI findings with radiographs and/or CT remains essential for accurate assessment of bone destruction, quality of periosteal reaction and presence of mineralisation.

Multiple symmetrical marrow lesions involving several tubular bones of the lower extremities should raise the suspicion of chronic multifocal osteomyelitis (CRMO), a non-pyogenic inflammatory disease that primarily affects bone [31]. The MRI appearance of CRMO is often quite characteristic, with osteolytic lesions based at the physis containing strongly enhancing inflammatory tissue, which are surrounded by oedema-like signal changes in the
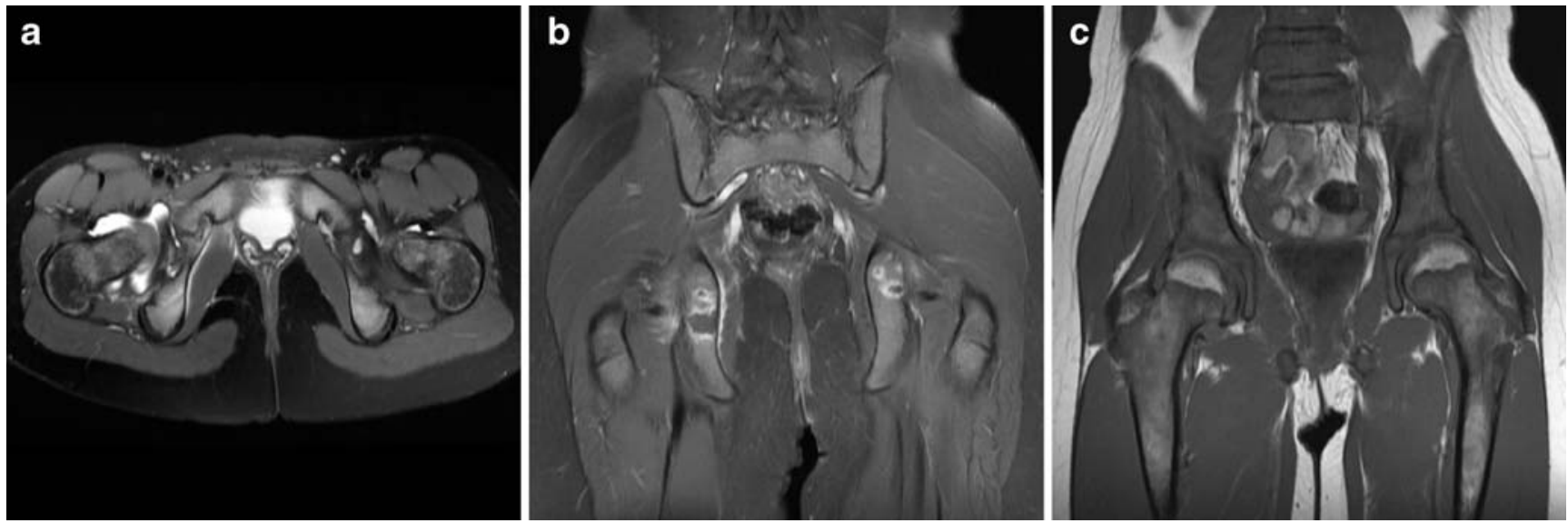

Fig. 6 MR images of a 10-year-old girl presenting with arthritis of the right hip. a Axial T2-W image with fat saturation shows a right hip effusion. b Coronal contrast-enhanced $\mathrm{T} 1-\mathrm{W}$ image with fat saturation shows multiple focal lesions with rim enhancement. These findings were misinterpreted as septic arthritis and multifocal bone abscesses. Arthrotomy revealed "purulent" joint fluid containing atypical white blood cells. The homogenously decreased bone marrow signal in the pelvis and lumbar vertebrae, isointense to muscle on T1-W image (c), should have suggested leukaemia clinically presenting as arthritis. Leukaemia was subsequently proven by bone marrow biopsy. The focal lesions seen in $\mathbf{b}$ probably represent areas of marrow infarction 
Fig. 7 A 12-year-old boy with elbow pain after skiing. Frontal (a) and lateral (b) radiographs show a lytic lesion in the radial metaphysis with cortical disruption and periosteal reaction. $\mathbf{c}$ Coronal STIR image shows inhomogeneous high marrow signal and a soft tissue mass (arrow). d Coronal T1-W image with fat saturation shows contrast enhancement of the soft tissue mass, marrow space and periosteal reaction (arrowhead). Biopsy revealed Langerhans cell histiocytosis
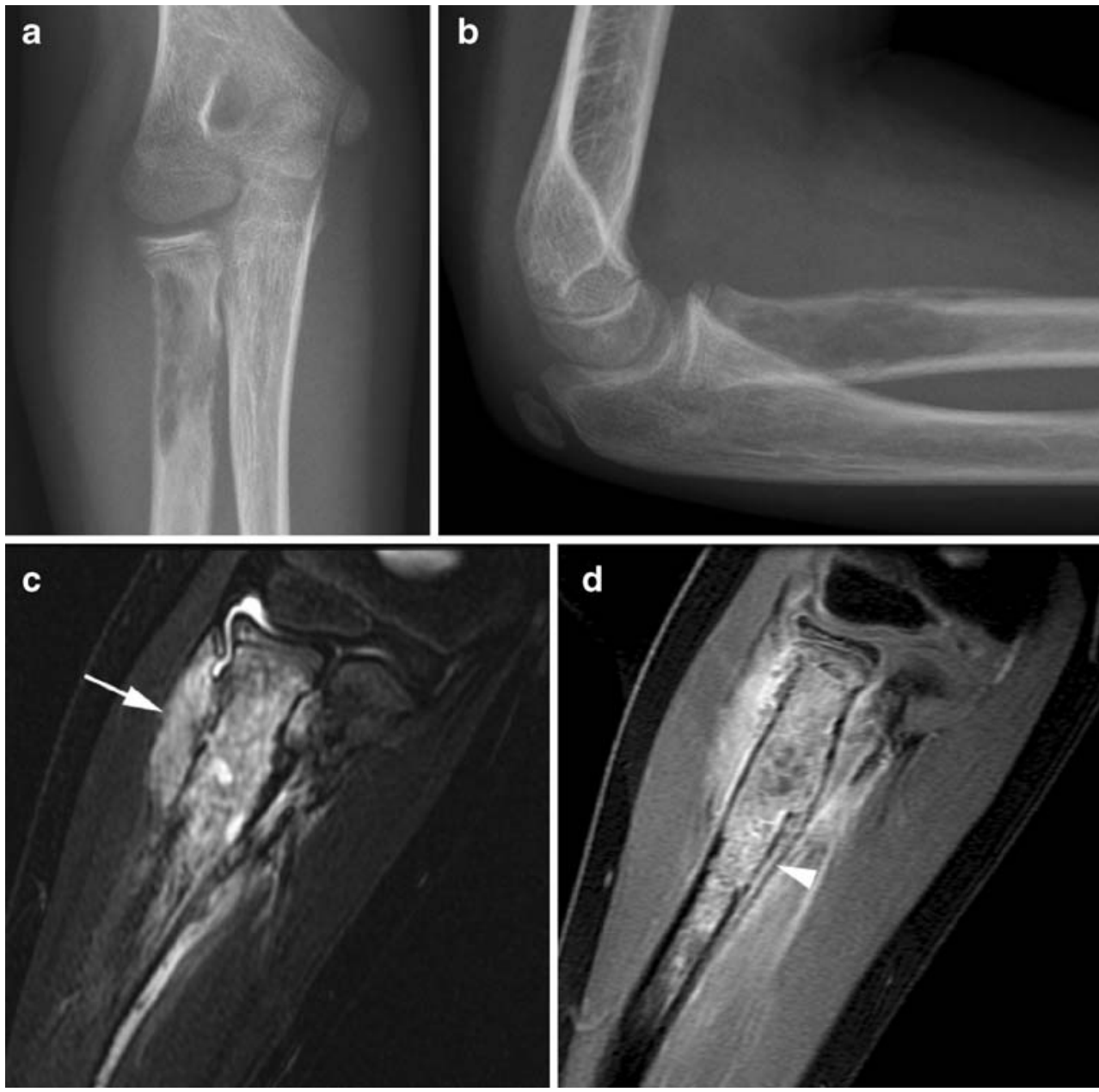

marrow and adjacent soft tissues (Fig. 8). Periosteal new bone formation can be seen in active lesions of CRMO, but abscesses and fistulous tracts are absent. CRMO is a diagnosis of exclusion, requiring biopsy to rule out infectious osteomyelitis and malignancy, which could present with a similar picture.
The extensive reactive marrow and soft tissue oedema that usually accompany an osteoid osteoma can be misleading, suggesting a more aggressive lesion such as acute osteomyelitis or malignant tumour. Conversely, oedematous changes may be minimal or absent, especially with medullary location of the osteoid osteoma (Fig. 9).
Fig. 8 MR images of a 15-yearold girl with CRMO. a Coronal T1-W and b STIR images show symmetrical involvement of both tibiae with characteristic lytic lesions based at the physes with adjacent oedema-like signal alteration
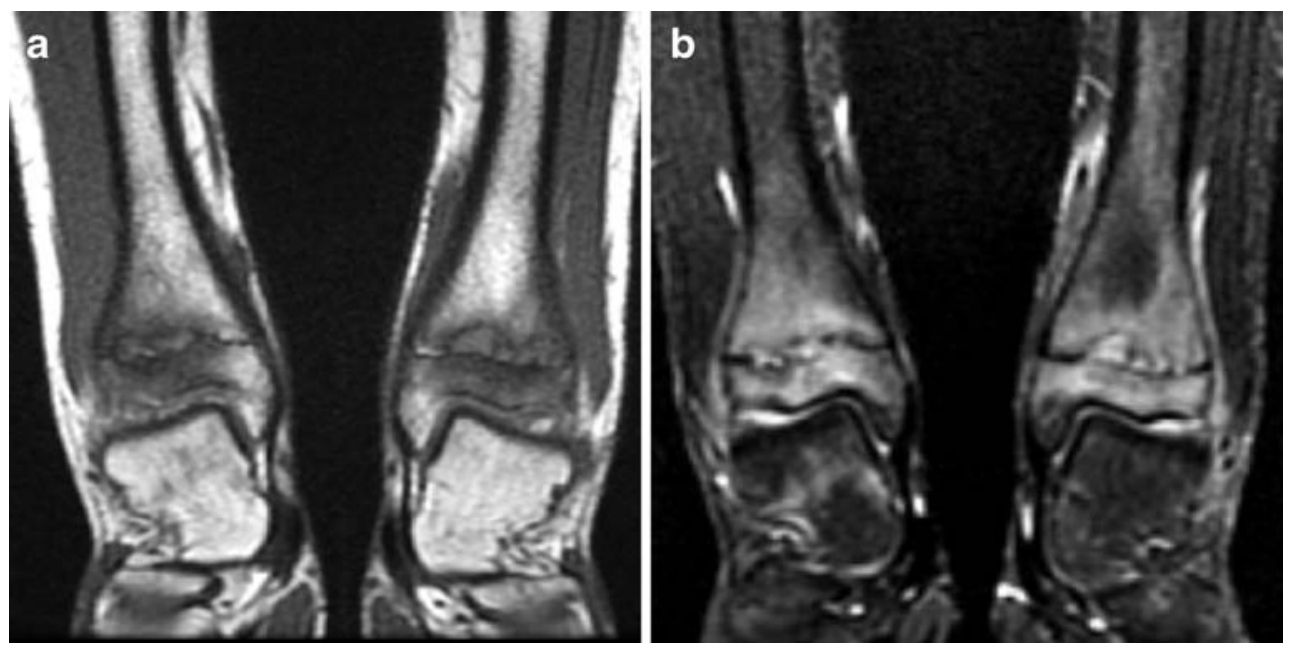

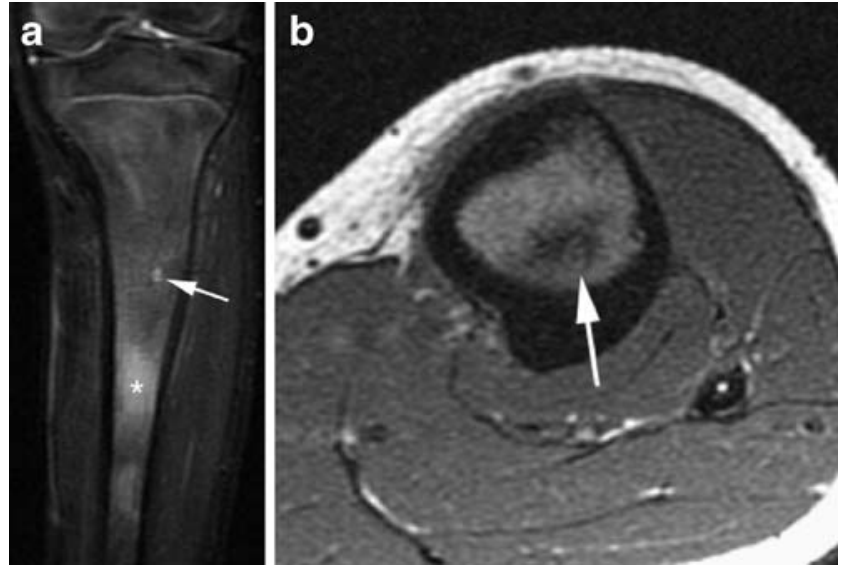

Fig. 9 MRI images of an 8-year-old boy with medullary osteoid osteoma. a Coronal STIR image shows minimal oedema-like marrow changes (asterisk) in the left tibia and a small hyperintense nidus (arrow). b T1-W axial image demonstrates a hypointense dot within the nidus (arrow) consistent with central calcification

With a careful search for an osteolytic nidus surrounded by bone proliferation, which would be diagnostic for an osteoid osteoma, false diagnoses can usually be avoided. However, a nidus is easily overlooked on MRI; when it is small and partly calcified it may be visible only on a single slice in a single plane, or it may not be visible at all [32]. Dynamic contrast-enhanced MRI can improve the conspicuity of a small hypervascular nidus [33], but ultimately thin-slice CT may be required for its localisation or exclusion.

Discrete hypointense lines or larger zones of sclerosis within an area of oedema-like marrow changes are signs of a fracture (Fig. 10); their identification can establish the diagnosis of stress or radiographically occult fractures. Oedema-like signal changes extending into tendinous attachments or a displaced apophysis indicate an avulsion injury.

Focal oedema-like marrow changes without any associated findings are non-specific, but can be due to pyogenic osteomyelitis (Fig. 2), CRMO, metastases, overuse injuries (stress reaction), and contusions (bone bruises) (Fig. 5). Correlation with the patient's history (trauma, sporting activities), clinical findings, laboratory tests (blood culture) and eventually biopsy is required for reaching a diagnosis.

Following the treatment of a malignant disease, one should be very cautious in the interpretation of newly detected marrow lesions. In our experience, such lesions more frequently represent hyperplastic red marrow, stress reaction or fracture of osteopenic bone, osteonecrosis following steroid therapy, or fibrotic scars, than recurrence and metastases.

\section{Soft tissue masses}

Some soft tissue masses can be diagnosed on MRI due to morphology, signal properties and degree of contrast enhancement (e.g. lipoma, vascular malformations, periarticular cysts). The frequently observed cavernous venous malformation typically presents as a well-defined hyperintense mass on T2-W images, with internal septations containing small hypointense phleboliths or thrombi, and showing slow inhomogeneous and often incomplete contrast enhancement. Unfortunately, soft tissue sarcomas may sometimes demonstrate a rather similar appearance (Fig. 11). The most common malignant soft tissue tumour mistaken for a benign lesion is synovial sarcoma, which can have a multilocular cystic appearance or resembles a haematoma with internal high signal on T1-W images [34]. Most soft tissue tumours have no characteristic features on MRI and it is usually not possible to distinguish between benign and malignant lesions. Tissue sampling is imperative for diagnosis because evaluation of size, margins, signal homogeneity and the extent of peritumoural oedema on MRI cannot reliably predict the nature of a solid soft tissue mass [35].
Fig. 10 MRI images of a 2year-old girl with radiographically occult fracture of the talus. a Sagittal T1-W and b STIR images show a hypointense fracture line in the head of the talus surrounded by oedema-like marrow signal changes
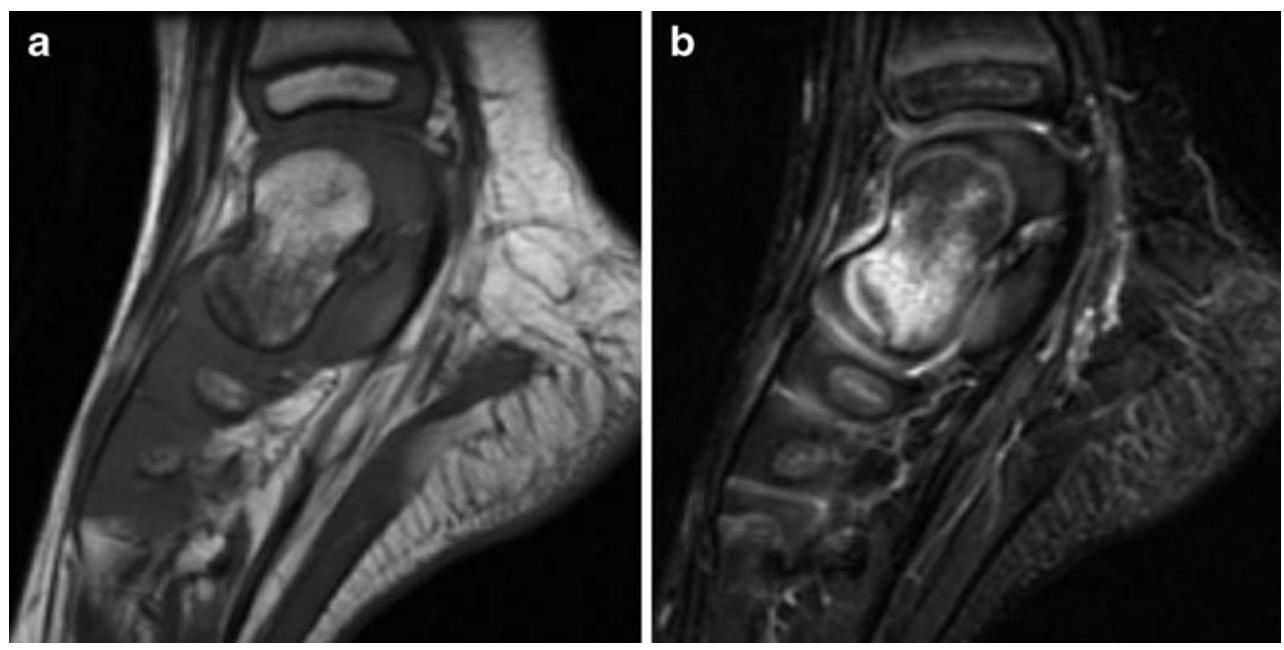
Fig. 11 MRI images of a 2year-old girl with a bluish mass at the left heel present since birth. a Sagittal STIR image shows a hyperintense soft tissue mass that appears to be septated and contains small hypointense thrombi (arrow). b Dynamic T1$\mathrm{W}$ gradient-echo image shows inhomogeneous and incomplete contrast enhancement. History and MRI findings were suggestive of a venous malformation, but biopsy at the time of attempted surgical size reduction revealed rhabdomyosarcoma
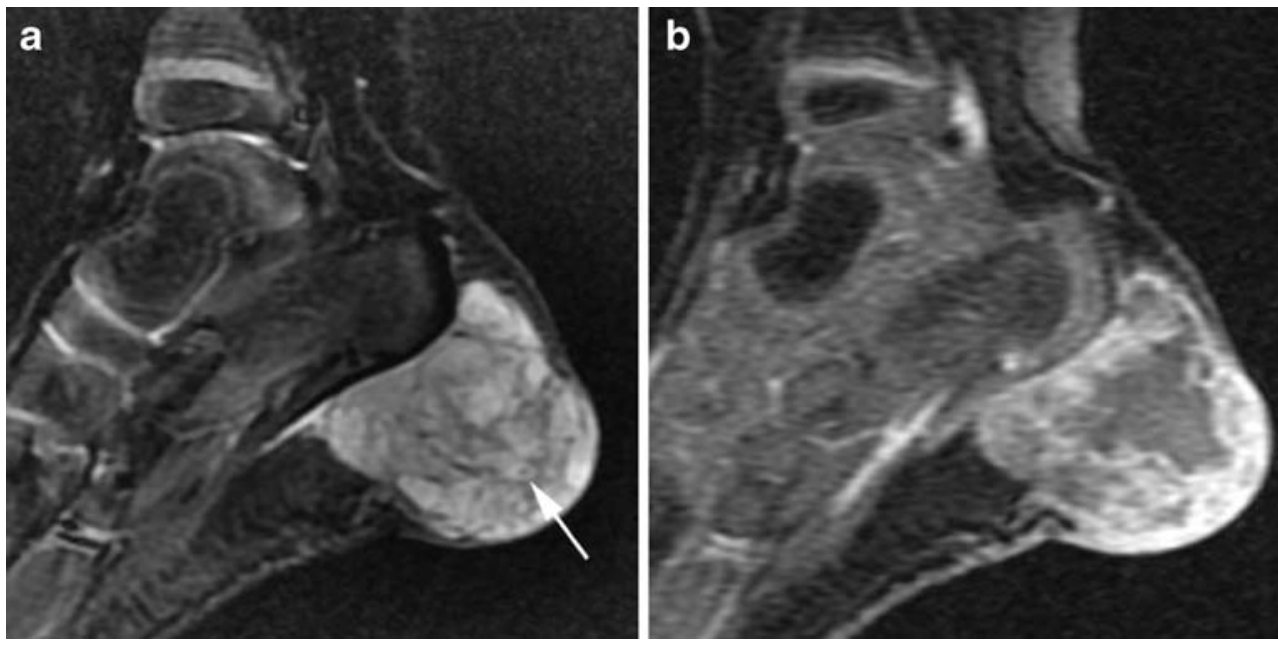

Myositis ossificans traumatica (MOT) is difficult to differentiate from infection or neoplasm on MRI, especially in the absence of a history of trauma. At an early stage of the disease, MOT can mimic an intramuscular abscess or necrotic tumour, with central fluid-fluid levels and rim enhancement. Early MOT can also resemble a solid tumour with ill-defined infiltrative margins and extensive surrounding soft tissue oedema (Fig. 12). In a child presenting with a palpable mass, swelling, pain and a clear history of trauma (direct blow or repeated minor trauma), MOT should be considered and can be proven by follow-up with different imaging modalities [36]. With maturation of the
Fig. 12 A 16-year-old boy with painful swelling of the left elbow following weight training. a Axial T2-W image shows an ill-defined hyperintense mass (arrow) within the flexor muscles, with extensive softtissue oedema and joint effusion (asterisks). b On T1-W imaging the mass is isointense to muscle while the T1-weighted image with fat saturation (c) shows inhomogeneous contrast enhancement. A soft tissue neoplasm was suspected, but biopsy revealed myositis ossificans. d Lateral radiograph obtained 4 weeks later confirms the diagnosis by demonstrating characteristic peripheral mineralisation
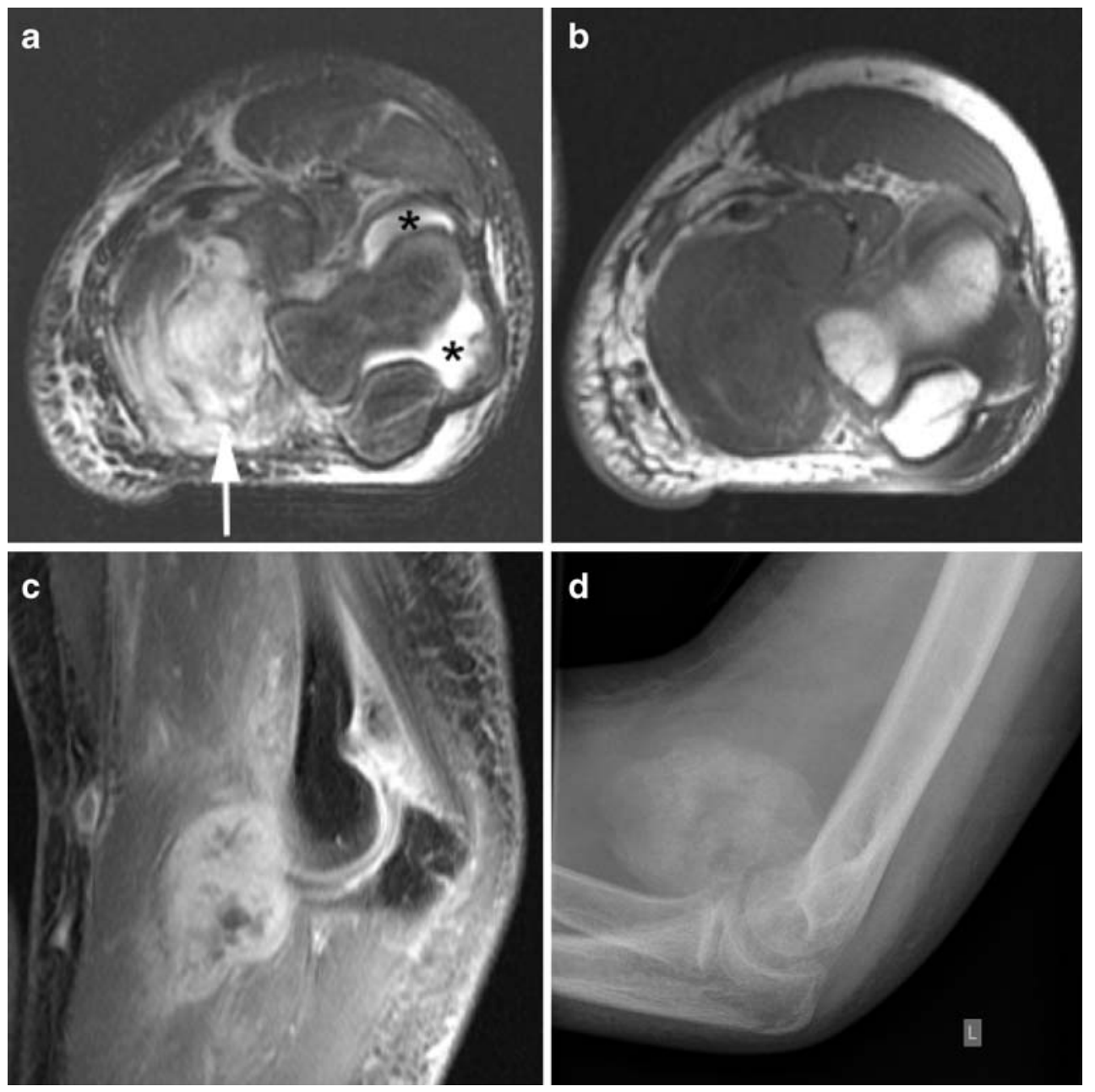
lesion, soft tissue oedema resolves and the now welldefined mass typically shows a rim of decreased signal intensity on all MRI sequences. This characteristic peripheral mineralisation pattern only appears after a few weeks and is better visualised on radiographs or CT.

Apophyseal avulsion injuries occur during periods of rapid growth when the physis is unfused. Acute avulsion injuries are usually easily detected as displaced bone fragments on radiographs. Subacute, healing or chronic avulsions can mimic an aggressive lesion with mixed lytic and sclerotic areas, new bone formation and a confusing appearance of a soft tissue mass on MRI. Familiarity with the common sites of avulsion injuries at muscle insertion points in the pelvis and other locations, as well as the history of the child's involvement in sports, help distinguish these traumatic lesions from more serious disease $[37,38]$.

\section{Conclusion}

In order to avoid potential pitfalls in the interpretation of musculoskeletal lesions in children, one has to be familiar with the pathologies, variants and changes occurring in the developing skeleton and soft tissues. As MRI findings may often be non-specific, correlation with radiographs and clinical context are mandatory for accurate interpretation. In many cases additional laboratory tests or tissue sampling are required to reach a definitive diagnosis.

Conflicts of interest The author declares that there are no conflicts of interest.

\section{References}

1. Slovis TL (2007) Caffey's pediatric diagnostic imaging. Mosby Elsevier, Oxford

2. Keats TE, Anderson MW (2006) Atlas of normal Roentgen variants that may simulate disease. Mosby Elsevier, Oxford

3. Freyschmidt J, Brossmann J, Wiens J et al (2003) Freyschmidt's "Köhler/Zimmer"-Borderlands of normal and early pathological findings in skeletal radiography. Thieme, Stuttgart

4. Araki Y, Tanaka H, Yamamoto H et al (1994) MRI of fibrous cortical defect of the femur. Radiat Med 12:93-98

5. Yamazaki T, Maruoka S, Takahashi S et al (1995) MR findings of avulsive cortical irregularity of the distal femur. Skeletal Radiol 24:43-46

6. Suh JS, Cho JH, Shin KH et al (1996) MR appearance of distal femoral cortical irregularity (cortical desmoid). J Comput Assist Tomogr 20:328-332

7. Posch TJ, Puckett ML (1998) Marrow MR signal abnormality associated with bilateral avulsive cortical irregularities in a gymnast. Skeletal Radiol 27:511-514

8. Jee WH, Choe BY, Kang HS et al (1998) Nonossifying fibroma: characteristics at MR imaging with pathologic correlation. Radiology 209:197-202
9. Lawson JP (1994) International Skeletal Society lecture in honor of Howard D. Dorfman. Clinically significant radiologic anatomic variants of the skeleton. AJR 163:249-255

10. Cawley KA, Dvorak AD, Wilmot MD (1983) Normal anatomic variant: scintigraphy of the ischiopubic synchondrosis. J Nucl Med 24:14-16

11. Kloiber R, Udjus K, McIntyre W et al (1988) The scintigraphic and radiographic appearance of the ischiopubic synchondroses in normal children and in osteomyelitis. Pediatr Radiol 18:57-61

12. Herneth AM, Philipp MO, Pretterklieber ML et al (2004) Asymmetric closure of ischiopubic synchondrosis in pediatric patients: correlation with foot dominance. AJR 182:361-365

13. Iqbal A, McKenna D, Hayes R et al (2004) Osteomyelitis of the ischiopubic synchondrosis: imaging findings. Skeletal Radiol $33: 176-180$

14. Vogler JB 3rd, Murphy WA (1988) Bone marrow imaging. Radiology 168:679-693

15. Moore SG, Bisset GS 3rd, Siegel MJ et al (1991) Pediatric musculoskeletal MR imaging. Radiology 179:345-360

16. Laor T, Jaramillo D (2009) MR imaging insights into skeletal maturation: what is normal? Radiology 250:28-38

17. Sze G, Bravo S, Baierl P et al (1991) Developing spinal column: gadolinium-enhanced MR imaging. Radiology 180:497-502

18. Dwek JR, Shapiro F, Laor T et al (1997) Normal gadoliniumenhanced MR images of the developing appendicular skeleton: part 2. Epiphyseal and metaphyseal marrow. AJR 169:191-196

19. Taccone A, Oddone M, Dell'Acqua AD et al (1995) MRI 'roadmap' of normal age-related bone marrow. II. Thorax, pelvis and extremities. Pediatr Radiol 25:596-606

20. Pal CR, Tasker AD, Ostlere SJ et al (1999) Heterogeneous signal in bone marrow on MRI of children's feet: a normal finding? Skeletal Radiol 28:274-278

21. Shabshin N, Schweitzer ME, Morrison WB et al (2006) Highsignal T2 changes of the bone marrow of the foot and ankle in children: red marrow or traumatic changes? Pediatr Radiol 36:670-676

22. Jaramillo D, Laor T, Hoffer FA et al (1991) Epiphyseal marrow in infancy: MR imaging. Radiology 180:809-812

23. Moore SG, Dawson KL (1990) Red and yellow marrow in the femur: age-related changes in appearance at MR imaging. Radiology 175:219-223

24. Zawin JK, Jaramillo D (1993) Conversion of bone marrow in the humerus, sternum, and clavicle: changes with age on MR images. Radiology 188:159-164

25. Waitches G, Zawin JK, Poznanski AK (1994) Sequence and rate of bone marrow conversion in the femora of children as seen on MR imaging: are accepted standards accurate? AJR 162:1399-1406

26. Sebag GH, Dubois J, Tabet M et al (1993) Pediatric spinal bone marrow: assessment of normal age-related changes in the MRI appearance. Pediatr Radiol 23:515-518

27. Dawson KL, Moore SG, Rowland JM (1992) Age-related marrow changes in the pelvis: MR and anatomic findings. Radiology 183:47-51

28. Zanetti M, Bruder E, Romero J et al (2000) Bone marrow oedema pattern in osteoarthritic knees: correlation between MR imaging and histologic findings. Radiology 215:835-840

29. Kan JH (2008) Major pitfalls in musculoskeletal imaging-MRI. Pediatr Radiol 38(Suppl 2):S251-S255

30. Azouz EM, Saigal G, Rodriguez MM et al (2005) Langerhans' cell histiocytosis: pathology, imaging and treatment of skeletal involvement. Pediatr Radiol 35:103-115

31. Jurik AG, Egund N (1997) MRI in chronic recurrent multifocal osteomyelitis. Skeletal Radiol 26:230-238

32. Davies M, Cassar-Pullicino VN, Davies AM et al (2002) The diagnostic accuracy of MR imaging in osteoid osteoma. Skeletal Radiol 31:559-569 
33. Liu PT, Chivers FS, Roberts CC et al (2003) Imaging of osteoid osteoma with dynamic gadolinium-enhanced MR imaging. Radiology 227:691-700

34. Nakanishi H, Araki N, Sawai Y et al (2003) Cystic synovial sarcomas: imaging features with clinical and histopathologic correlation. Skeletal Radiol 32:701-707

35. Moulton JS, Blebea JS, Dunco DM et al (1995) MR imaging of soft-tissue masses: diagnostic efficacy and value of distinguishing between benign and malignant lesions. AJR 164:1191-1199
36. Gindele A, Schwamborn D, Tsironis K et al (2000) Myositis ossificans traumatica in young children: report of three cases and review of the literature. Pediatr Radiol 30:451-459

37. Stevens MA, El-Khoury GY, Kathol MH et al (1999) Imaging features of avulsion injuries. Radiographics 19:655-672

38. Rossi F, Dragoni S (2001) Acute avulsion fractures of the pelvis in adolescent competitive athletes: prevalence, location and sports distribution of 203 cases collected. Skeletal Radiol 30:127-131 